\title{
Psoriasiform Keratosis - Case report
}

Carla Andréa Avelar Pires ${ }^{1}$

Carla do Socorro Silva do Nascimento ${ }^{1}$

Mario Fernando Ribeiro de Miranda ${ }^{2}$
Brena Andrade de Sousa ${ }^{1}$

Ana Thais Machado Moutinho

Francisca Regina Oliveira Carneiro ${ }^{1}$

Abstract: Psoriasiform Keratosis is a rare clinic entity. The etiopathogenesis remains unknown and the disease is characterized by a solitary, scaly or keratotic papule, or plaque mainly located on the extremities. Histopathological features closely resemble those of psoriasis. We report the case of a 70-year-old woman presenting a solitary and asymptomatic keratotic plaque, located on the back of the left leg, unresponsive to topical corticosteroids. We performed an excisional biopsy and histopathology was consistent with psoriasiform keratosis. Keywords: Acanthoma; Keratosis; Psoriasis

\section{INTRODUCTION}

Psoriasiform keratosis was described as a clinicopathological disorder by Walsh et al in $2007 .{ }^{1}$ It is a rare clinical entity characterized by a solitary papule or plaque with scaly or keratotic surface, located mainly in the lower limbs. ${ }^{1,2,3}$ The clinical lesion mimics seborrheic keratosis, actinic keratosis, or squamous cell carcinoma and histopathology resembles psoriasis, with abnormal differentiation, keratinocyte hyperproliferation and infiltration of inflammatory components. ${ }^{1,4}$

Etiopathogenesis is still unknown, but some authors believe that it is classified in the group of cutaneous acanthomas including acantholytic acanthoma, epidermolytic acanthoma, clear cell acanthoma, verrucous dyskeratoma, seborrheic keratosis and lichen planus-like keratosis. ${ }^{1}$ This report aims to present an uncommon case of psoriasiform keratosis on the left lower limb.

\section{CASE REPORT}

This is a seventy year-old female patient, retired, born and raised in the city of Inhangapi (state of Pará). She complained of the appearance of a single and asymptomatic lesion on the posterior surface of the left leg, three months ago (Figure 1). She had used neomycin cream and betamethasone dipropionate ointment without any improvement. Histopathological

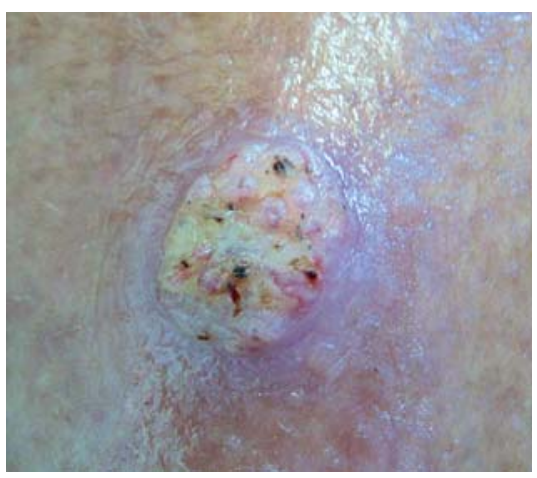

FIGURE 1:

Single, keratotic plaque, located on the posterior surface of the left leg

examination revealed sections of skin showing epidermis with pronounced psoriasiform hyperplasia associated with hyperkeratosis, intra-corneal neutrophilic aggregates, vacuolated and pale aspect of more superficial keratinocytes and suprapapillary epidermal atrophy. In the upper dermal compartment, ectasic/congested vessels and perivascular and diffuse lymphocytic inflammatory infiltrate were observed. Absence of atypias was noted (Figures 2 and 3). Mycological examination and culture for fungi were negative. Excisional biopsy of the lesion was performed, without recurrence until present.

\footnotetext{
Received on 01.02.2013.

Approved by the Advisory Board and accepted for publication on 25.02.2013.

* Work performed at the Dermatology Service at Universidade do Estado do Pará (UEPA) - Belém (PA), Brazil

Conflict of interest: None

Financial funding: None

Universidade do Estado do Pará (UEPA) - Belém (PA), Brazil.

Universidade Federal do Pará (UFPA) - Belém (PA), Brazil.

(C2014 by Anais Brasileiros de Dermatologia
} 


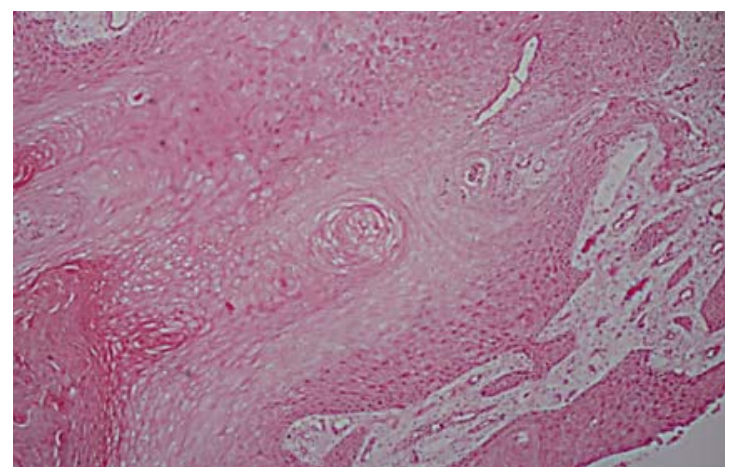

FIGURE 2: Histopathology: pronounced psoriasiform hyperplasia, pale aspect of the more superficial keratinocytes and suprapapillary epidermal atrophy

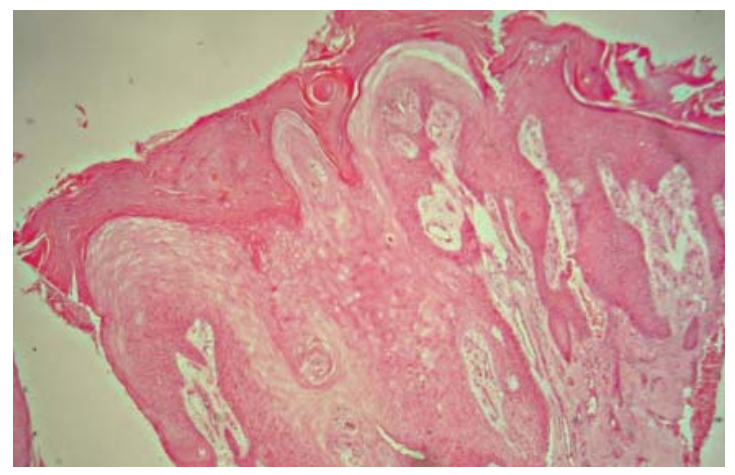

FIGURE 3: Histopathology: epidermis showing hyperkeratosis, parakeratosis, hypergranulosis, irregular epidermal hyperplasia and intracorneal collections of neutrophils. Superior dermis shows vascular dilation and perivascular lymphocytic infiltrate

\section{DISCUSSION}

Psoriasiform keratosis is a benign proliferation of the epidermis usually seen in older people, of both sexes. Etiology and prevalence are unknown. ${ }^{1,3}$

Most lesions are found in the legs, unlike psoriasis, which is characterized by often affecting areas such as elbows, knees, and scalp. The lack of evidence of psoriasis elsewhere, absence of family history and lack of response to corticosteroids exclude the diagnosis of psoriasis..$^{2,3,4}$

In histopathological analysis, psoriasiform keratosis resembles psoriasis, and includes regular and irregular acanthosis, sometimes with focal parakeratosis, neutrophils on the corneal layer, intraepidermal spongiform pustules, small dilated vessels in the papillary dermis, and perivascular lymphocytic infiltrate. ${ }^{1,5}$

Our patient presented involvement of the posterior surface of the left leg, like most cases reported in the literature and had histopathology results similar to those of psoriasis.

Differential diagnoses include conditions such as verrucous psoriasis, clear cell acanthoma, and various forms of actinic keratosis. ${ }^{5}$

Like the case reported by Carbone et al, topical steroids therapy was ineffective in our patient, justifying the fact that psoriasiform keratosis may be a sui generis epithelial proliferation with histological features of psoriasis, but without any relation to it. Therefore, other approaches are necessary, such as surgical excision. ${ }^{2}$

\section{REFERENCES}

1. Sezer E, Böer A, Falk T. Identification of Human Papilloma Virus Type 6 in Psoriasiform Keratosis. Am J Dermatopathol. 2010;32:492-4.

2. Carbone A, De Simone C, Valenzano F, Amerio P, Massi G. Psoriasiform keratosis. Eur J Dermatol. 2009;19:631-2.

3. Mutasim DF. Psoriasiform Keratosis: A Lesion Mimicking Psoriasis. Am J Dermatopathol. 2007;29:482-4.

4. Arruda LHF, Campbell GAM, Takahashi MDF. Psoríase. Psoriasis. An Bras Dermatol. 2001;76:141-67.

5. Walsh SN, Hurt MA, Santa Cruz DJ. Psoriasiform Keratosis. Am J Dermatopathol. 2007;29:137-40.

\author{
MAILING ADDRESS: \\ Brena Andrade de Sousa \\ Travessa Perebebuí, 2623 - Marco \\ 66087-670 - Belém PA \\ Brazil \\ E-mail:brenasousa@gmail.com
}

How to cite this article: Pires CAA, Sousa BA, Nascimento CSS, Moutinho ATM, Miranda MFR, Carneiro FRO. Psoriasiform Keratosis - Case report. An Bras Dermatol. 2014;89(2):318-9. 